\title{
Estudo da atividade antibacteriana dos monoterpenos timol e carvacrol contra cepas de Escherichia coli produtoras de $\beta$-lactamases de amplo espectro
}

\section{Study of the antibacterial activity of thymol and carvacrol monoterpenes against strains of Escherichia coli producing extended-spectrum $\beta$-lactamases}

Dijaci Santos de Lima', Jade Cardôso Lima', Raqueline Maiara Costa Bezerra Calvacanti', Bernadete Helena Cavalcanti dos Santos², Igara Oliveira Lima

' Universidade Federal de Campina Grande, Centro de Educação e Saúde, Unidade Acadêmica de Saúde, Cuité, Paraíba, Brasil

2 Universidade Federal da Paraíba, Centro de Ciências da Saúde, Departamento de Ciências Farmacêuticas, João Pessoa, Paraíba, Brasil

${ }^{3}$ Universidade Federal de Campina Grande, Centro de Educação e Saúde, Unidade Acadêmica de Saúde, João Pessoa, Paraíba, Brasil

\begin{abstract}
RESUMO
OBJETIVOS: Estudar a atividade antimicrobiana dos fitoconstituintes timol e carvacrol contras cepas de Escherichia coli produtoras de $\beta$-lactamases de amplo espectro (ESBL) e determinar a concentração inibitória mínima (CIM) do timol e do carvacrol. MATERIAIS E MÉTODOS: Os experimentos foram realizados no Laboratório de Microbiologia (J11) no Centro de Educação e Saúde da Universidade Federal de Campina Grande, estado da Paraíba, Brasil. Para a determinação da CIM dos fitoconstituintes selecionados, foram utilizadas cepas bacterianas de $E$. coli ESBL (C-18, C-21, C-20, C-24, C-25, 24, 65) oriundas de pacientes ambulatoriais e a técnica de microdiluição em placa de 96 orifícios por meio da diluição seriada a uma razão de dois, reservando a última coluna para o controle do crescimento dos microrganismos. RESULTADOS: A CIM do carvacrol apresentou concentrações de $64 \mu \mathrm{g} / \mathrm{mL}$ para a cepa 65 e $128 \mu \mathrm{g} / \mathrm{mL}$ para as cepas C-18, C-20, C-21, C-24, C-25 e 24. Já frente à ação do timol, a CIM foi determinada como: $1.024 \mu \mathrm{g} / \mathrm{mL}$ para a cepa C-25; $512 \mu \mathrm{g} / \mathrm{mL}$ para as cepas C-18, C-21, 24 e 65; $256 \mu \mathrm{g} / \mathrm{mL}$ para a cepa C-24; e $128 \mu \mathrm{g} / \mathrm{mL}$ para a cepa C-20. CONCLUSÃO: Por meio dos experimentos, pôde-se afirmar que os fitoconstituintes carvacrol e timol exercem atividade bacteriostática sobre as cepas de E. coli.
\end{abstract}

Palavras-chave: Escherichia coli; Betalactamases; Terpenos.

\begin{abstract}
OBJECTIVES: To study the antimicrobial activity of thymol and carvacrol phytochemicals against strains of Escherichia coli producing extended-spectrum $\beta$-lactamases (ESBL) and to determine the minimum inhibitory concentration (MIC) of thymol and carvacrol. MATERIALS AND METHODS: The experiments were carried out at the Microbiology Laboratory (J11) at the Education and Health Center of the Universidade Federal de Campina Grande, Paraíba State, Brazil. In order to determine the MIC of the selected phytocompounds, bacterial strains of E. coli ESBL (C-18, C-21, C-20, C-24, C-25, 24, 65) from outpatients were used, and microdilution technique in 96-hole reaction plate by two-fold serial dilution, reserving the last column for growth control of microorganisms. RESULTS: MIC of carvacrol showed concentrations of $64 \mu \mathrm{g} / \mathrm{mL}$ for strain 65 and $128 \mu \mathrm{g} / \mathrm{mL}$ for strains C-18, C-20, C-21, C-24, C-25, and 24. In the thymol action, MIC was determined as: 1,024 $\mu \mathrm{g} / \mathrm{mL}$ for strain C-25; $512 \mu \mathrm{g} / \mathrm{mL}$ for C-18, C-21, 24, and 65; $256 \mu \mathrm{g} / \mathrm{mL}$ for C-24; and $128 \mu \mathrm{g} / \mathrm{mL}$ for strain C-20. CONCLUSION: Through the experiments it was possible to affirm that the phytocompounds carvacrol and thymol have bacteriostatic effects on E. coli strains.
\end{abstract}

Keywords: Escherichia coli; Beta-lactamases; Terpenes.

\footnotetext{
Correspondência / Correspondence:

Dijaci Santos de Lima

Universidade Federal de Campina Grande, Centro de Educação e Saúde, Unidade Acadêmica de Saúde

Olho D'água da Bica, s/n - CEP: 58175-000 - Cuité, Paraíba, Brasil

E-mail: dijaci.lima@hotmail.com
} 


\section{INTRODUÇÃO}

Escherichia coli habita normalmente 0 intestino da maioria dos animais, incluindo seres humanos. Algumas cepas de E. coli podem provocar uma ampla variedade de doenças intestinais e extraintestinais, tais como diarreia, infecções do trato urinário, septicemia e meningite neonatal'. Apresenta fácil manuseio em laboratório com alta facilidade em seu cultivo, é bastante conhecida em relação ao quesito genético, e pode ser empregada como indicador de contaminação fecal da água².

As $\beta$-lactamases de amplo espectro (extendedspectrum $\beta$-lactamase - ESBL) são enzimas capazes de hidrolisar o grupo farmacofórico (anel betalactâmico) dos antibióticos betalactâmicos, como cefalosporinas, penicilinas e monobactâmicos, sendo consideradas uma forma terapêutica primária para o controle de infecções. Essas enzimas são codificadas em genes presentes principalmente nos plasmídeos, transpósons e integrons, elementos esses que apresentam capacidade de carregar outros tipos de genes de resistência, conferindo assim a esse microrganismo resistência às demais classes de fármacos ${ }^{3}$.

Por muito tempo, as bactérias Gram negativas eram controladas por meio do tratamento com cefalosporinas; entretanto, essa realidade vem mudando, uma vez que têm surgido cepas que expressam a ESBL, sendo essa um dos principais mecanismos de resistência na família Enterobacteriaceae $e^{4,5,6}$.

Devido ao constante aumento dos meios de resistência bacterianos, sendo uma considerável parte oriunda da automedicação, o combate à resistência microbiana acaba se tornando uma grande preocupação da Organização Mundial da Saúde. São necessários investimentos não só na área da saúde, mas também, de suma importância, na área da educação para o desenvolvimento de diferentes formas de controle e prevenção da multirresistência bacteriana $^{7}$.

○ surgimento da resistência por parte dos microrganismos é derivado de mutações genéticas espontâneas ou oriundas de estímulos e recombinação de genes, resultando em uma ampla variedade genética, adequando-os à sobrevida em ocasiões adversas; dessas, as mais comumente enfrentadas por eles são a ação de antimicrobianos ou a resistência adquirida pelo contato com organismos já resistentes. Essas alterações são possíveis pelo genoma bacteriano ser bem dinâmico, com as atividades de defesa e transferência gênica sendo desempenhadas por estruturas móveis, como integrons e plasmídios, que possibilitam, então, a ampla troca e modificação de dados genéticos ${ }^{8,9}$.

A detecção de espécies portadoras das ESBL se faz necessária para identificar essas cepas e definir a terapêutica apropriada para um tratamento eficaz ${ }^{10}$. Entretanto, há falhas na terapêutica medicamentosa e os programas de pesquisa têm propiciado a descoberta e a viabilidade de alternativas terapêuticas frente aos mecanismos de resistência. Dessa forma, produtos naturais têm demonstrado grande eficiência nas infecções bacterianas; sendo assim, não é surpresa - fato de existir uma grande variedade de drogas oriundas de tais produtos ${ }^{11}$. Nessa perspectiva, pesquisas têm sido desenvolvidas no intuito de investigar novos fármacos com ação antimicrobiana ou, ainda, moléculas que modulam a atividade de antibióticos $^{11,12}$.

Os óleos essenciais e os terpenos têm apresentado diversas atividades biológicas, dentre elas ações antimicrobianas, quando testados na presença de bactérias como E. coli. $O$ carvacrol e o timol são isômeros e têm sido apontados por alguns autores como moléculas promissoras no estudo de alternativas terapêuticas para o tratamento de infecções ${ }^{12,13,14}$.

Os terpenos correspondem a uma classe de substâncias químicas derivadas do metabolismo secundário dos vegetais, cuja origem biossintética deriva de unidades de isopreno $\left(\mathrm{C}_{5} \mathrm{H}_{8}\right)$, existindo, em sua maior parte, na forma de estrutura cíclica, sendo formado a partir do ácido mevalônico ${ }^{15}$. Estudos têm mostrado que os óleos essenciais do orégano e do tomilho apresentam atividade antimicrobiana e possuem carvacrol, timol e eugenol como constituintes majoritários, sendo que a concentração desses fitoconstituintes pode variar de região para região ${ }^{16}$.

○ timol, também conhecido por 2-isopropil-5metilfenol, é um monoterpeno, sendo isomérico com carvacrol; é extraído como uma substância aromática, de coloração cristalina branca e de odor agradável, pouco solúvel em água, mas sendo extremamente solúvel em alguns solventes orgânicos; apresenta $\mathrm{pH}$ neutro. $\bigcirc$ timol também apresenta características alcalinas em soluções aquosas devido à desprotonação do fenol ${ }^{17}$.

O carvacrol, ou 2-metil-5-1-metiletilfenol, é também um monoterpeno e apresenta característica picante e cheiro brando de orégano. Devido a essas características e ao cheiro agradável, ele é utilizado como aditivo alimentar ${ }^{18}$. Acredita-se ser a ruptura da membrana das bactérias a causa das propriedades antimicrobianas do carvacrol ${ }^{19}$.

Diante dessas premissas, o objetivo deste trabalho foi investigar a ação antibacteriana do timol e do carvacrol contra cepas de E. coli ESBL.

\section{MATERIAIS E MÉTODOS}

\section{LOCAL DE TRABALHO}

Os experimentos foram realizados no Laboratório de Microbiologia (J11) do Centro de Educação e Saúde da Universidade Federal de Campina Grande, estado da Paraíba, Brasil.

\section{CEPAS BACTERIANAS}

Foram utilizadas cepas bacterianas de E. coli ESBL (C-18, C-21, C-20, C-24, C-25, 24, 65), cedidas pela farmacêutica Bernadete Helena Cavalcanti Santos 
(Laboratório de Microbiologia Clínica, Departamento de Ciências Farmacêuticas, Universidade Federal da Paraíba), oriundas de pacientes ambulatoriais.

\section{FITOCONSTITUINTES}

Os fitoconstituintes testados foram o timol e o carvacrol (Sigma Aldrich).

\section{DETERMINAÇÃO DA CONCENTRAÇÃO INIBITÓRIA MÍNIMA}

A determinação da concentração inibitória mínima (CIM) dos fitoconstituintes selecionados foi realizada pela técnica da microdiluição em placa de 96 orifícios e fundo em "U" para cada uma das cepas. Por meio da dilvição seriada a uma razão de 2 , foram obtidas as concentrações de 1.024 a $1 \mu \mathrm{g} / \mathrm{mL}$, efetuadas na própria placa, da coluna 1 à 11. A última coluna (12) foi reservada para o controle de crescimento do microrganismo (Caldo Mueller-Hinton, sem o produto testado). Ainda foram realizados os seguintes controles: para avaliar a toxicidade do veículo, a esterilidade do meio e a viabilidade do inóculo bacteriano $20,21,22$

Em seguida, adicionou-se $10 \mu \mathrm{L}$ do inóculo (bactéria a 0,5 McFarland) em cada uma das cavidades. $\bigcirc$ ensaio foi realizado em triplicata e incubado a $37{ }^{\circ} \mathrm{C}$ no período de $24 \mathrm{~h}$ para E. coli ESBL. Após o tempo de incubação adequado, foram adicionados $20 \mu \mathrm{L}$ de resazurina a $1 \%$ (SIGMA), indicador colorimétrico de óxido-redução para bactérias; procedeu-se a leitura, visualmente, pela ausência ou presença de crescimento do microrganismo, observando-se a mudança da coloração da solução de azul para rosa/vermelho, resultante do crescimento do microrganismo.
Portanto, foi determinada como CIM a menor concentração do produto capaz de inibir o crescimento do microrganismo ensaiado, verificado por uma não mudança da coloração do corante indicador $20,21,22$.

\section{RESULTADOS}

Os resultados referentes à ação bacteriostática do carvacrol e timol contra cepas de E. coli ESBL encontram-se na tabela 1, em que se determinou a CIM do carvacrol como sendo $64 \mu \mathrm{g} / \mathrm{mL}$ para a cepa 65 e $128 \mu \mathrm{g} / \mathrm{mL}$ para as cepas C-18, C-20, C-21, C-24, C-25 e 24. Já para o timol, a CIM foi determinada como $1.024 \mu \mathrm{g} / \mathrm{mL}$ para a cepa C-25; $512 \mu \mathrm{g} / \mathrm{mL}$ para as cepas C-18, C-21, 24 e 65; $256 \mu \mathrm{g} / \mathrm{mL}$ para a cepa C-24; e $128 \mu \mathrm{g} / \mathrm{mL}$ para a cepa C-20.

\section{DISCUSSÃO}

Óleos essenciais que contêm constituintes como o carvacrol e o timol vêm sendo observados como agentes antibacterianos, antivirais, antidiabéticos e antioxidantes em doenças cardiovasculares. Tais fitoconstituintes são relatados como potenciais agentes antimicrobianos, principalmente frente às bactérias Gram positivas ${ }^{23}$. Entretanto, eles se mostraram ativos contra a maioria das cepas testadas, em especial o carvacrol.

Estudos realizados a partir de extratos produzidos de folhas adultas de Ficus benjamina confirmaram a atividade antibacteriana dos constituintes dessa planta e obtiveram uma CIM de $1.024 \mu \mathrm{g} / \mathrm{mL}$ contra cepas de E. coli ${ }^{24}$, corroborando o resultado obtido na cepa E. coli 25, quando testada a atividade do timol no presente trabalho.

Tabela 1 - CIM em $\mu \mathrm{g} / \mathrm{mL}$ do carvacrol e timol contra cepas de E. coli

\begin{tabular}{|c|c|c|c|c|c|c|c|}
\hline & $\begin{array}{c}\text { E. coli } \\
\text { C-18 }\end{array}$ & $\begin{array}{l}\text { E. coli } \\
\text { C-20 }\end{array}$ & $\begin{array}{l}\text { E. coli } \\
\text { C-21 }\end{array}$ & $\begin{array}{l}\text { E. coli } \\
\text { C-24 }\end{array}$ & $\begin{array}{l}\text { E. coli } \\
\text { C-25 }\end{array}$ & $\begin{array}{c}\text { E. coli } \\
24\end{array}$ & $\begin{array}{c}\text { E. coli } \\
65\end{array}$ \\
\hline $2.048 \mu \mathrm{g} / \mathrm{mL}$ & $C(-) \mathrm{T}(-)$ & $C(-) T(-)$ & $C(-) T(-)$ & $C(-) T(-)$ & $C(-) T(-)$ & $C(-) T(-)$ & $C(-) T(-)$ \\
\hline $1.024 \mu \mathrm{g} / \mathrm{mL}$ & $C(-) \mathrm{T}(-)$ & $C(-) \mathrm{T}(-)$ & $C(-) T(-)$ & $C(-) T(-)$ & $C(-) T(-)$ & $C(-) \mathrm{T}(-)$ & $C(-) T(-)$ \\
\hline $512 \mu \mathrm{g} / \mathrm{mL}$ & $C(-) \mathrm{T}(-)$ & $C(-) \mathrm{T}(-)$ & $C(-) T(-)$ & $C(-) T(-)$ & $C(-) T(+)$ & $C(-) \mathrm{T}(-)$ & $C(-) T(-)$ \\
\hline $256 \mu \mathrm{g} / \mathrm{mL}$ & $C(-) T(+)$ & $C(-) \mathrm{T}(-)$ & $C(-) T(+)$ & $C(-) T(-)$ & $C(-) T(+)$ & $C(-) T(+)$ & $C(-) T(+)$ \\
\hline $128 \mu \mathrm{g} / \mathrm{mL}$ & $C(-) T(+)$ & $C(-) T(-)$ & $C(-) \mathrm{T}(+)$ & $C(-) \mathrm{T}(+)$ & $C(-) T(+)$ & $C(-) \mathrm{T}(+)$ & $C(-) T(+)$ \\
\hline $64 \mu \mathrm{g} / \mathrm{mL}$ & $C(+) \mathrm{T}(+)$ & $\mathrm{C}(+) \mathrm{T}(+)$ & $C(+) T(+)$ & $C(+) \mathrm{T}(+)$ & $\mathrm{C}(+) \mathrm{T}(+)$ & $\mathrm{C}(+) \mathrm{T}(+)$ & $C(-) T(+)$ \\
\hline $32 \mu \mathrm{g} / \mathrm{mL}$ & $C(+) T(+)$ & $C(+) T(+)$ & $C(+) T(+)$ & $C(+) \mathrm{T}(+)$ & $C(+) T(+)$ & $\mathrm{C}(+) \mathrm{T}(+)$ & $C(+) \mathrm{T}(+)$ \\
\hline $16 \mu \mathrm{g} / \mathrm{mL}$ & $\mathrm{C}(+) \mathrm{T}(+)$ & $\mathrm{C}(+) \mathrm{T}(+)$ & $\mathrm{C}(+) \mathrm{T}(+)$ & $\mathrm{C}(+) \mathrm{T}(+)$ & $C(+) T(+)$ & $\mathrm{C}(+) \mathrm{T}(+)$ & $\mathrm{C}(+) \mathrm{T}(+)$ \\
\hline $8 \mu \mathrm{g} / \mathrm{mL}$ & $\mathrm{C}(+) \mathrm{T}(+)$ & $\mathrm{C}(+) \mathrm{T}(+)$ & $\mathrm{C}(+) \mathrm{T}(+)$ & $\mathrm{C}(+) \mathrm{T}(+)$ & $\mathrm{C}(+) \mathrm{T}(+)$ & $\mathrm{C}(+) \mathrm{T}(+)$ & $\mathrm{C}(+) \mathrm{T}(+)$ \\
\hline $4 \mu \mathrm{g} / \mathrm{mL}$ & $C(+) T(+)$ & $C(+) T(+)$ & $C(+) \mathrm{T}(+)$ & $C(+) T(+)$ & $C(+) T(+)$ & $\mathrm{C}(+) \mathrm{T}(+)$ & $C(+) T(+)$ \\
\hline $2 \mu \mathrm{g} / \mathrm{mL}$ & $C(+) T(+)$ & $C(+) T(+)$ & $C(+) \mathrm{T}(+)$ & $C(+) T(+)$ & $C(+) T(+)$ & $\mathrm{C}(+) \mathrm{T}(+)$ & $C(+) T(+)$ \\
\hline $1 \mu \mathrm{g} / \mathrm{mL}$ & $\mathrm{C}(+) \mathrm{T}(+)$ & $C(+) T(+)$ & $\mathrm{C}(+) \mathrm{T}(+)$ & $C(+) T(+)$ & $C(+) T(+)$ & $C(+) T(+)$ & $C(+) \mathrm{T}(+)$ \\
\hline
\end{tabular}

(+): Crescimento bacteriano visível; (-): Não houve crescimento bacteriano visível; C: Carvacrol; T: Timol. 
De acordo com a literatura, a ação biológica de ambos os fitoconstituintes pode ser atribuída à indução nas deformações da membrana celular que ambos causam, alterando a sua permeabilidade. Além disso, é possível que núcleos aromáticos presentes em sua estrutura e que contêm um grupo polar possam estabelecer ligações de hidrogênio com enzimas microbianas, 0 que provavelmente favoreceria a atividade antimicrobiana ${ }^{25,26}$. Também se pode atribuir a atividade antimicrobiana desses fitoconstituintes à sua suposta atividade pró-oxidante, dessa forma podendo torná-los excelentes agentes antimicrobianos quando em pequenas concentrações ${ }^{13}$.

\section{CONCLUSÃO}

Por meio dos experimentos pôde-se afirmar que os fitoconstituintes carvacrol e timol exercem atividade bacteriostática frente às cepas de $E$. coli.

\section{REFERÊNCIAS}

1 Ørskov F, Ørskov I. Escherichia coli serotyping and disease in man and animals. Can J Microbiol. 1992 Jul;38(7):699-704.

2 Cordeiro ACS, Leite SGF, Dezotti M. Inativação por oxidação fotocatalítica de Escherichia coli e Pseudomonas sp. Quim Nova. 2004 set-out;27(5):689-94.

3 Silva KC, Lincopan N. Epidemiologia das betalactamases de espectro estendido no Brasil: impacto clínico e implicações para o agronegócio. J Bras Patol Med Lab. 2012 abr;48(3):91-9.

4 Lago A, Fuentefria SR, Fuentefria DP. Enterobactérias produtoras de ESBL em Passo Fundo, Estado do Rio Grande do Sul, Brasil. Rev Soc Bras Med Trop. 2010 jul-ago;43(4):430-4.

5 Rawat D, Nair D. Extended-spectrum $\beta$-lactamases in Gram negative bacteria. J Glob Infect Dis. 2010 Sep;2(3):263-74

6 Rath S, Dubey D, Sahu MC, Padhy RN. Surveillance of ESBL producing multidrug resistant Escherichia coli in a teaching hospital in India. Asian Pac J Trop Dis. 2014 Apr;4(2):140-9.

7 Wollheim C. Epidemiologia molecular de Escherichia coli e Klebsiella spp produtoras de beta-lactamase de espectro ampliado [dissertação]. Caxias do Sul (RS): Universidade de Caxias do Sul, Instituto de Biotecnologia; 2009. 170 p.

8 Nijsten R, London N, van den Bogaard A, Stobberingh E. Antibiotic resistance of Enterobacteriaceae isolated from the faecal flora of fattening pigs. Vet Q. 1993 Dec;15(4):152-7.

9 Smith HW. Clinical problems of preventive medicine. Antibiotic-resistant bacteria in animals: the dangers to human health. Br Vet J. 1974 Mar-Apr;130(0): 1 10-9.

10 Fernebro J. Fighting bacterial infections - Future treatment options. Drug Resist Updat. 2011 Apr;14(2): 125-39.

11 Coutinho HDM, Costa JGM, Lima EO, FalcãoSilva VS, Siqueira-Júnior JP. In vitro interference of Momordica charantia in the resistance to aminoglycosides. Pharm Biol. 2009;47(1 1):1056-9.
12 Veras HNH, Rodrigues FFG, Botelho MA, Menezes IRA, Coutinho HDM, Costa JGM. Enhancement of aminoglycosides and $\beta$-lactams antibiotic activity by essential oil of Lippia sidoides Cham. and the Thymol. Arab J Chem. In Press 2013.

13 Bakkali F, Averbeck S, Averbeck D, Idaomar M. Biological effects of essential oils: a review. Food Chem Toxicol. 2008 Feb;46(2):446-75.

14 Özkan A, Erdoğan A. A comparative evaluation of antioxidant and anticancer activity of essential oil from Origanum onites (Lamiaceae) and its two major phenolic components. Turk J Biol. $2011 ; 35: 735-42$.

15 Robbers, JE, Speedie MK, Tyler VE. Farmacognosia e biotecnologia. São Paulo: Premier; 1997. 372 p.

16 Cosentino S, Tuberoso CIG, Pisano B, Satta $M$, Mascia V, Arzedi E, et al. In-vitro antimicrobial activity and chemical composition of Sardinian Thymus essential oils. Lett Appl Microbiol. 1999 Aug;29(2):130-5.

17 Medicines and Healthcare products Regulatory Agency (UK). British Pharmacopoeia: Index, BP 2009 [Internet]. London: MHRA; 2009 [cited 2015 Aug 10]. Available from: http://www. pharmacopoeia.co.uk/pdf/2009_index.pdf.

18 Ultee A, Slump RA, Steging G, Smid EJ. Antimicrobial activity of carvacrol toward Bacillus cereus on rice. J Food Prot. 2000 May;63(5):620-4.

19 Cristani M, D'Arrigo M, Mandalari G, Castelli $F$, Sarpietro MG, Micieli $D$, et al. Interaction of four monoterpenes contained in essential oils with model membranes: implications for their antibacterial activity. J Agric Food Chem. 2007 Jul;55(15):6300-8.

20 National Committee for Clinical Laboratory Standards. Reference method for broth dilution antifungal susceptibility testing of yeasts; approved standard — second edition. Wayne: NCCLS; 2002. 29 p. (NCCLS document M27-A2; vol. 22, no. 15).

21 Sarker SD, Nahar L, Kumarasamy Y. Microtitre plate-based antibacterial assay incorporating resazurin as an indicator of cell growth, and its application in the in vitro antibacterial screening of phytochemicals. Methods. 2007 Aug;42(4):321-4. 
22 Hussain Al, Anwar F, Nigam PS, Sarker SD, Moore JE, Rao JR, et al. Antibacterial activity of some Lamiaceae essential oils using resazurin as an indicator of cell growth. Food Sci Technol. 2011 May;44(4): 1 199-206.

23 Edris AE. Pharmaceutical and therapeutic potentials of essential oils and their individual volatile constituents: a review. Phytother Res. 2007 Apr;21 (4):308-23.

24 Reschke A, Marques LM, Mayworm MAS. Atividade antibacteriana de Ficus benjamina L. (Moraceae). Rev Bras PI Med. 2007;9(2):67-70.
25 Lambert RJW, Skandamis PN, Coote PJ, Nychas GJE. A study of the minimum inhibitory concentration and mode of action of oregano essencial oil, thymol and carvacrol. J Appl Microbiol. 2001 Sep;91(3):453-62.

26 Ultee A, Bennik MHJ, Moezelaar R. The phenolic hydroxyl group of carvacrol is essential for action against the food-borne pathogen Bacillus cereus. Appl Environ Microbiol. 2002 Apr;68(4):1561-8.

Recebido em / Received: 10/3/2016 Aceito em / Accepted: 13/10/2016 